\title{
Optical Scattering Properties of Intralipid Phantom in Presence of Encapsulated Microbubbles
}

\author{
Homa Assadi, ${ }^{1}$ Raffi Karshafian, ${ }^{1}$ and Alexandre Douplik ${ }^{1,2}$ \\ ${ }^{1}$ Department of Physics, Ryerson University, 350 Victoria Street, Toronto, ON, Canada M5B 2K3 \\ ${ }^{2}$ Erlangen Graduate School in Advanced Optical Technologies (SAOT), Friedrich-Alexander-Universität Erlangen-Nürnberg, \\ Paul Gordan Strasse 6, D-91052 Erlangen, Germany
}

Correspondence should be addressed to Homa Assadi; homa.assadi@ryerson.ca

Received 20 December 2013; Revised 15 February 2014; Accepted 2 March 2014; Published 2 April 2014

Academic Editor: Victor Loschenov

Copyright (C) 2014 Homa Assadi et al. This is an open access article distributed under the Creative Commons Attribution License, which permits unrestricted use, distribution, and reproduction in any medium, provided the original work is properly cited.

\begin{abstract}
In imaging, contrast agents are utilized to enhance sensitivity and specificity of diagnostic modalities. In ultrasound imaging, microbubbles (MBs) - a gas-core shell-encapsulated agent-are used clinically as contrast agents. The working hypothesis of this study is that microbubbles can be employed as an intravascular contrast agent in optical imaging systems. In this work, the interaction of light and microbubbles in a turbid medium (intralipid) was investigated, particularly, the effect of MBs on the reduced scattering and absorption coefficients. Diffuse reflectance (DR) and total transmittance (TT) measurements of highly scattering intralipid suspension (0.5-5\%) were measured using spectroscopic integrating sphere system in the absence and presence of Definity microbubbles. The optical properties were computed using the inverse adding doubling (IAD) software. The presence of microbubbles increased DR and decreased TT of intralipid phantoms. In the presence of MBs ( $0.5 \%$ volume concentration), the reflectance of the intralipid phantom increased from $35 \%$ to $100 \%$. The reduced scattering coefficient increased significantly (30\%) indicating potential use of MBs as optical contrast agents in light based modalities.
\end{abstract}

\section{Introduction}

Microbubbles (MBs), a gas-core shell-encapsulated bubble of 1-5 micrometers in diameter, are utilized clinically in diagnostic ultrasound imaging as a contrast agent [1]. The gas of microbubbles is usually comprised of perfluorocarbon, nitrogen, or sulfur hexafluoride gas. The shell of microbubbles normally comprises of lipids, proteins, and polymers [1] and its thickness is in nanometer range $(60-200 \mathrm{~nm})$ [2]. Microbubbles behave as an intravascular contrast agent; they remain within the circulation. They generally have a short lifetime; generally MBs disappear within a few minutes following an intravenous injection [3]. Encapsulated microbubbles potentially can be used as optical contrast agents since MBs contribute to the media refraction index mismatch. The presence of MBs in tissue can cause refractive index mismatches and potentially allow the MBs to be distinguished from its surrounding media. The gas and shell of microbubbles will only affect the real part of refractive index. There is no considerable optical absorption and only refraction occurs as a result of light-microbubble interactions which may increase light scattering and subsequently increase optical contrast. The size of microbubbles is on the order of a wavelength, and therefore the main optical effect of light-MB interaction is Mie scattering [4].

Light based modalities are nonionizing methods with significant potential for noninvasive, portable, and costeffective medical diagnostics [5]. However, at visible and near IR wavelengths $(400-1200 \mathrm{~nm})$ light scattering contrast is limited between adjacent tissues [6]. Although, for example, morphological differences between normal and malignant tissues can be obvious at later stages of tumor development, it is challenging to detect early-stage tumors or tumors that are optically similar to surrounding normal tissue [7]. This can be potentially improved by contrast agents to differentiate normal tissue from the pathological lesions exploiting MBs as contrast agents in similar manner as for US imaging contrast enhancement [8]. 
The goal of this work is to study the effect of MBs on light propagation in a turbid media. Intralipid was used to mimic biological tissue scattering properties. In biomedical photonics, light propagation is an important process in both diagnostic and therapeutic applications [9]. Light propagation through any media is determined by its optical properties, which are wavelength dependent [10]. The intrinsic optical properties of tissues are absorption coefficient, $\mu_{a}$, scattering coefficient, $\mu_{s}$, scattering anisotropy, $g$, reduced scattering coefficient, $\mu_{s}^{\prime}$, and refraction index $n$ [11]. Typically, tissue is a turbid media and the optical reduced scattering coefficient for soft tissues is in the range $10-100[1 / \mathrm{cm}]$. Intralipid is turbid and has no strong absorption in the visible region and is often used as a tissue mimicking phantom medium [12].

In this work, various concentrations of intralipid were used to demonstrate a diffuse reflectance similar to soft tissues with known scattering coefficients. The absorption and scattering coefficients are obtained from measured transmittance and reflectance using an inverse adding doubling method (IAD) based on radiative transfer theory. IAD is an accurate solution of the radiative transport equation for all albedos, all optical depths, and all phase functions and can be applied to any medium for which the radiative transport equation is valid [13]. In this study, the effect of microbubbles mixed with intralipid upon the optical properties of the intralipid solutions at various concentrations is studied using the integrating sphere spectroscopy measurements and inverse adding doubling algorithm.

\section{Materials and Method}

\subsection{Materials}

2.1.1. Intralipid Phantom. Intralipid (IL) phantoms with varying concentrations of IL $0.5 \%, 1 \%, 1.5 \%, 2 \%, 3 \%$, and $5 \%(\mathrm{v} / \mathrm{v}$ : volume concentration) of the original $20 \%$ stock solution (Sigma-Aldrich, Canada) were used to mimic the scattering properties of biological tissue. The intralipid 20\%, according to the manufacturer, is made of $20 \%$ soybean oil, $1.2 \%$ egg yolk phospholipids, $2.25 \%$ glycerin, and water. The glycerin is dissolved into individual molecules in the water and does not scatter light. The difference in the refractive index of waterglycerin solution from that of pure water has no measurable influence on the scattering parameters [4]. The dilutions of IL can yield wavelength-dependent reduced scattering coefficients $\left(\mu_{s}^{\prime}\right)$ in the range from 5.7 to $26.7[1 / \mathrm{cm}]$ that are similar to values reported for tissues [14].

2.1.2. Microbubbles. Definity (perflutren lipid microspheres, Lantheus Medical Imaging, Billerica, MA), an FDA approved commercially available ultrasound microbubble contrast agent, was used in this study. Definity microbubbles with the mean size of $1.2 \pm 0.5 \mu \mathrm{m}$, measured using a Coulter Counter Multisizer (Beckman Coulter, USA) (Figure 3), were added to the intralipid phantom at $2.5 \% \mathrm{v} / \mathrm{v}$ (volume concentration). The microbubble concentration is comparable to values used in ex vivo ultrasound experiments [15]. Definity has a concentration of $1.2 * 10^{10}$ bubbles $/ \mathrm{mL}$.

\subsection{Methods}

2.2.1. Spectroscopy. Double beam integrating sphere technique (UV-VIS-NIR Spectrophotometer Shimadzu UV-3600, Japan) was used to determine the total transmittance (TT) and diffuse reflectance (DR) of intralipid phantoms with and without microbubbles. Visible and near infrared wavelengths $(300-850 \mathrm{~nm})$ and $8 \mathrm{~nm}$ slit width measurements were performed [16]. The wavelength accuracy in UV/VIS region: $\pm 0.2 \mathrm{~nm}$ and NIR region: $\pm 0.8 \mathrm{~nm}$.

The intralipid phantom was placed in $2 \mathrm{~mm}$ light path quartz cuvettes (Starna Cells Inc., USA). Cuvette dimensions were $45 \mathrm{~mm} \times 4.5 \mathrm{~mm} \times 12.5 \mathrm{~mm}$ (considered as an infinite in the direction perpendicular to the incident beam) and held at the integrating sphere sample port. The experimental conditions satisfy the requirement of optically thick $(\tau \sim 1)$ tissue sample which ensures the validity of the multiple scattering [10]. In diffusive regime, $\mu_{s}^{\prime}$ completely describes the light scattering profile.

2.2.2. Analysis. Inverse adding doubling (IAD) software (developed by Prahl, 2011) program was used to compute the optical properties of the samples [17]. The settings of the IAD program were sample index of refraction (1.47: phantom (the scattering particles of intralipid are soybean oil. The refractive index of soybean oil is $n=1.47$ and was considered as the refractive index of the sample [18])), index of refraction of the top and bottom slides (1.5138: BK-7), sample thickness ( $2 \mathrm{~mm})$, illumination beam diameter $(7.1365 \mathrm{~mm})$, reflectivity of the reflectance calibration standard (0.98), and number of spheres used during each measurement (1). The properties of reflectance measurement sphere were sphere diameter $(60 \mathrm{~mm})$, sample port diameter $(18.74 \mathrm{~mm})$, entrance port diameter $(16.73 \mathrm{~mm})$, detector port diameter (13.12 mm), and reflectivity of the sphere wall (0.98). The properties of transmittance measurement sphere were sphere diameter $(60 \mathrm{~mm})$, sample port diameter $(16.73 \mathrm{~mm})$, entrance port diameter (0.00), detector port diameter (13.12), and reflectivity of the sphere wall (0.98).

Measured DR and TT were used as input for the IAD software to extract $\mu_{a}$ and $\mu_{s}^{\prime}$ as a function of wavelength [11]. The inverse adding doubling algorithm initially guesses the intrinsic optical properties set, comparing the expected observables with those measured by the integrating sphere system and then iteratively approaching the final set of intrinsic parameters minimizing the deviation from the measured values of TT and DR [17]. The IAD technique is shown to calculate optical properties within ten percent of the true optical properties for all sample thicknesses greater than one optical depth $\tau[10]$.

2.3. Experiments. The diffuse reflectance (DR) and total transmittance (TT) were measured at visible and near infrared wavelengths (from 300 to $850 \mathrm{~nm}$ ) for intralipid phantoms $(0.5 \%, 1 \%, 1.5 \%, 2 \%, 3 \%$, and $5 \%)$ before and after adding microbubbles. $2 \mathrm{~mm}$ light path quartz cuvettes (Starna Cells Inc., USA) were used to hold the samples at the integrating sphere sample ports. This ensured the validity 

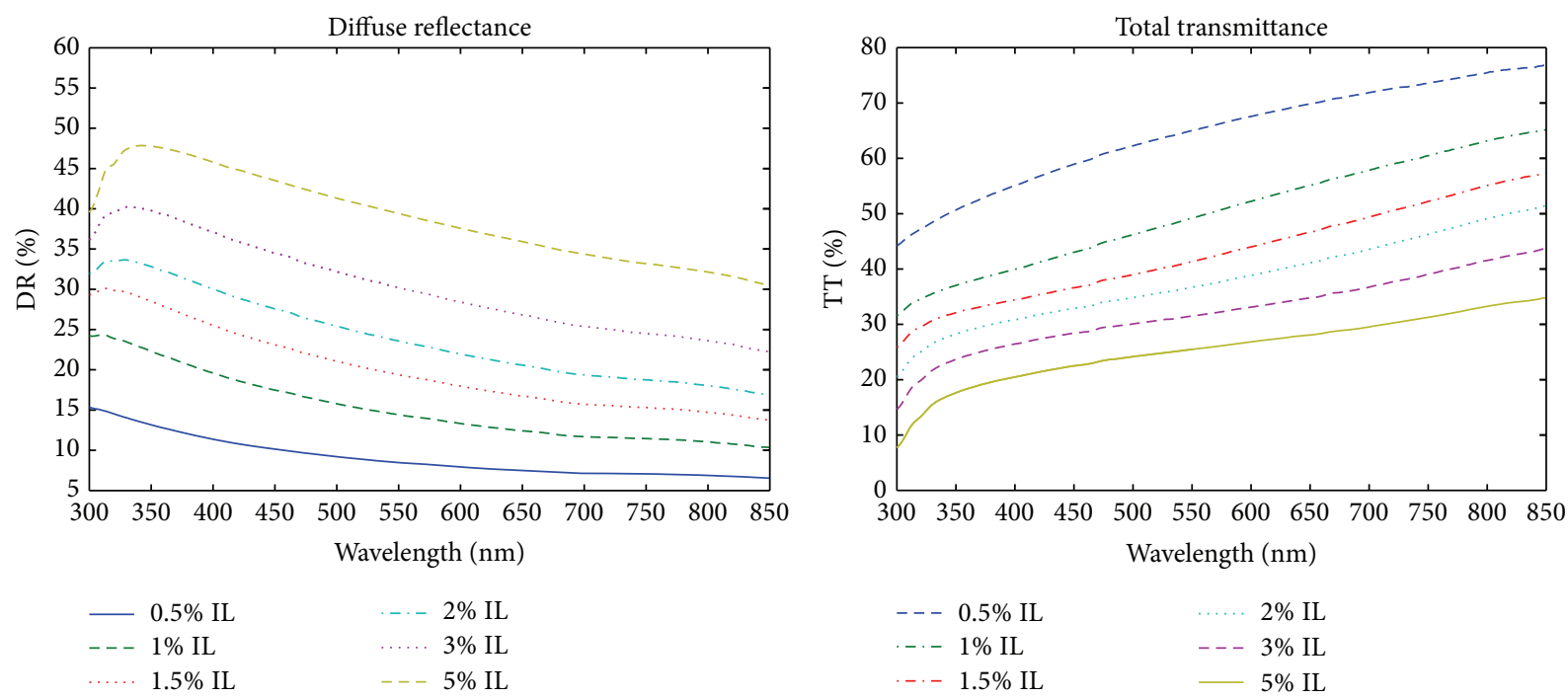

FIGURE 1: Diffuse reflectance and total transmittance for all concentrations of intralipid.

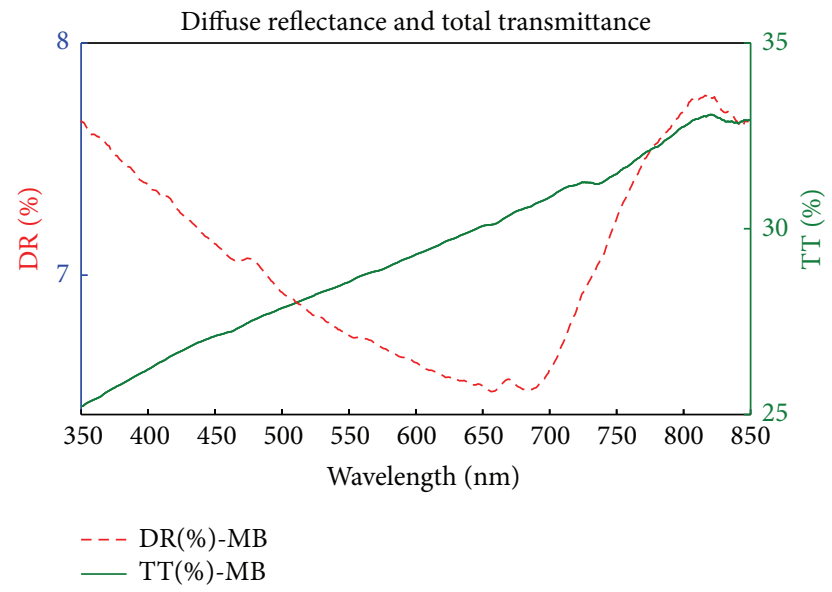

FIGURE 2: Diffuse reflectance and total transmittance for microbubbles diluted in deionized water (without intralipid).

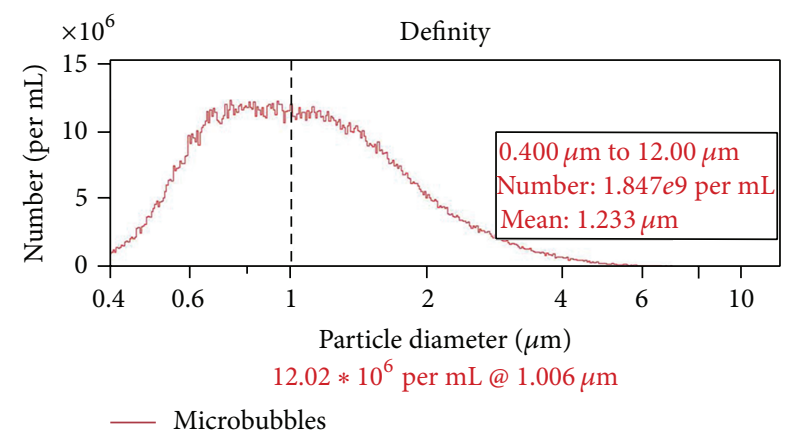

FIGURE 3: Coulter Counter Multisizer 4 measurements of the size of Definity microbubbles. of the multiple scattering for optically thick $(\tau \sim 1)$ tissue samples [10]. Thus, the $\mu_{s}^{\prime}$ describes the light scattering profile completely. Spectra in the range from 300 to $850 \mathrm{~nm}$ at a spectral resolution of $0.1 \mathrm{~nm}$ were obtained for (1) intralipid solutions only (no MBs) and (2) intralipid solutions with MBs. The MB volume ratio was 1:40 for all measurements which is in the range of ex vivo ultrasound studies [15].

\section{Results and Discussion}

3.1. Reflectance and Transmittance Spectra. The diffuse reflectance (DR) and total transmittance (TT) of intralipid phantoms at varying concentrations in the absence of MBs are shown in Figure 1. DR increases and TT decreases with increasing intralipid concentration, as expected. The DR and TT for microbubbles alone diluted in deionized water are shown in Figure 2. The size distribution of Definity microbubbles is shown in Figure 3.

The DR and TT of phantoms in the absence and presence of microbubbles at varying concentrations of intralipids are shown in Figures 4, 5, 6, and 7; the spectra with the addition of microbubbles are shown in solid line. The DR increases and TT decreases in the presence of microbubbles for all intralipid concentrations. For example, in the $0.5 \%$ intralipid concentration phantom, DR increased by $35-100 \%$ and TT decreased by $25-40 \%$ in the presence of MBs (Figure 4). In addition, the effect of microbubbles depended on the wavelength. At longer wavelengths, the effect of microbubbles on reflectance signal is decreased, whereas the effect is increased for total transmittance. Furthermore, the reflectance and transmittance spectra depended on intralipid concentration. With increasing intralipid concentration, the effect of MB on DR and TT was significantly reduced. At intralipid concentrations higher than 1.5\%, DR and TT with and without microbubbles appear to be similar (Figure 7). 

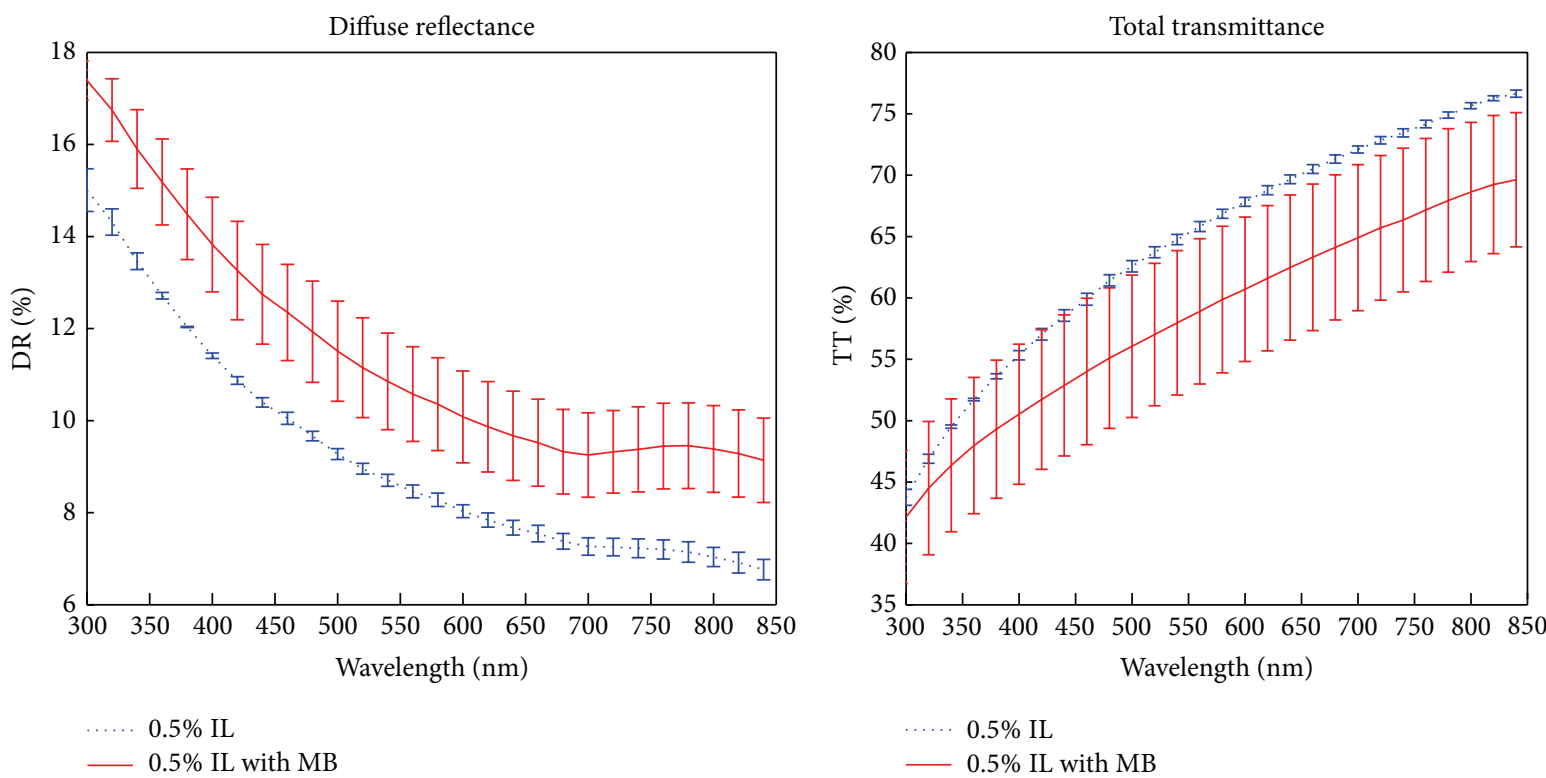

FIGURE 4: Diffuse reflectance (DR) and total transmittance (TT) of diluted intralipid (IL), $0.5 \%$ concentration, before and after adding Definity microbubbles.
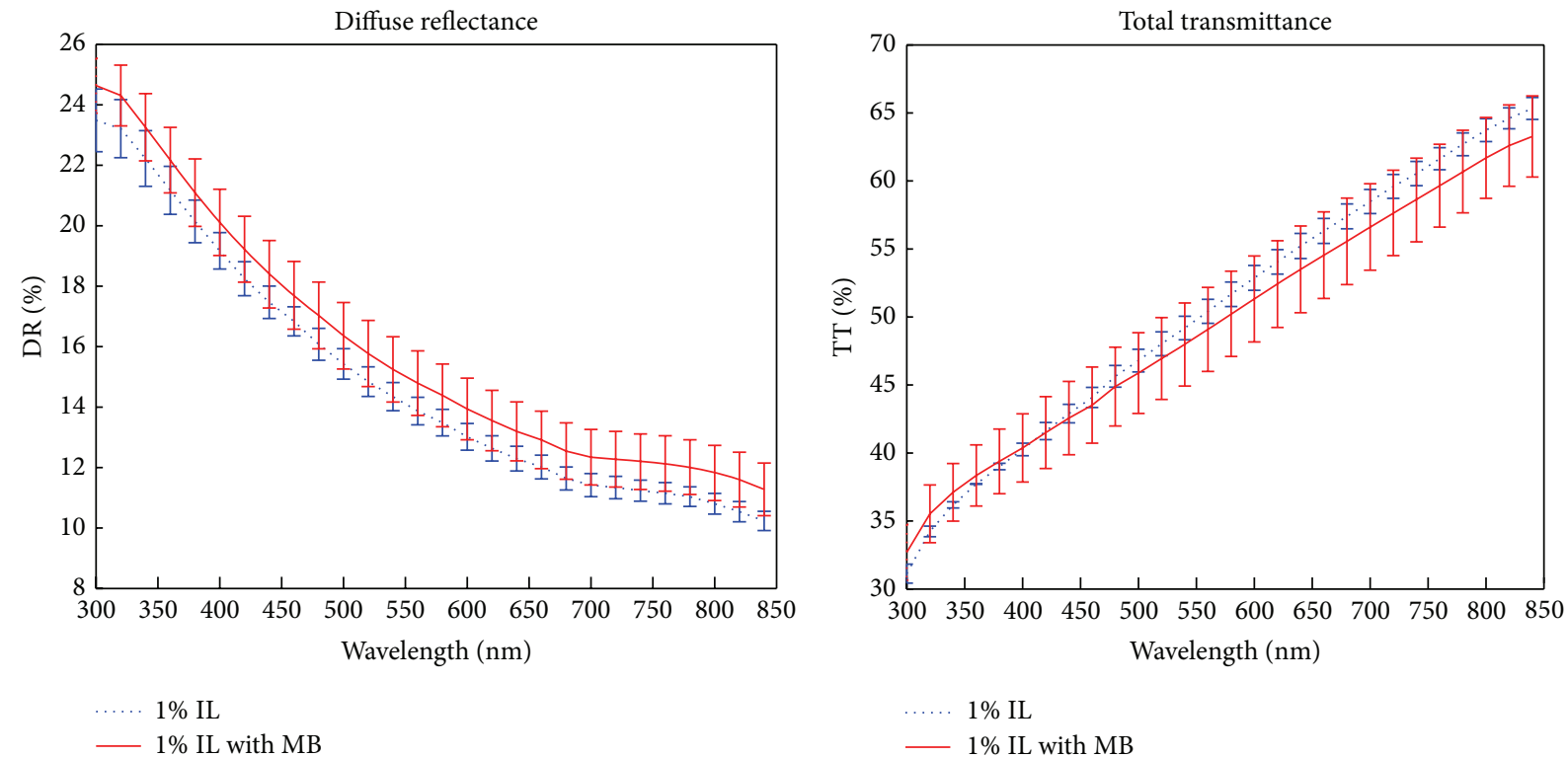

FIGURE 5: Diffuse reflectance (DR) and total transmittance (TT) of diluted intralipid (IL), $1 \%$ concentration, before and after adding Definity microbubbles.

3.2. Spectra of Intrinsic Optical Properties in Presence of Microbubbles. The optical properties (reduced scattering and absorption coefficients) for intralipid phantoms $(0.5 \%$ to $5.0 \%)$ and microbubbles only in deionized water are shown in Figures 8 and 9, respectively. The reduced scattering and the absorption coefficients increase with increasing intralipid concentration (Figure 8). The characteristics of the spectra were similar; the amplitude in the reduced scattering spectrum decreased with intralipid concentration. Microbubbles act as optical scatterers in deionized water (Figure 9).

The computed reduced scattering and absorption coefficients as a function of wavelength for various concentrations of IL in the absence and presence of microbubbles are shown in Figures 10, 11, 12, and 13. The altered $\mu_{a}$ and $\mu_{s}^{\prime}$ 

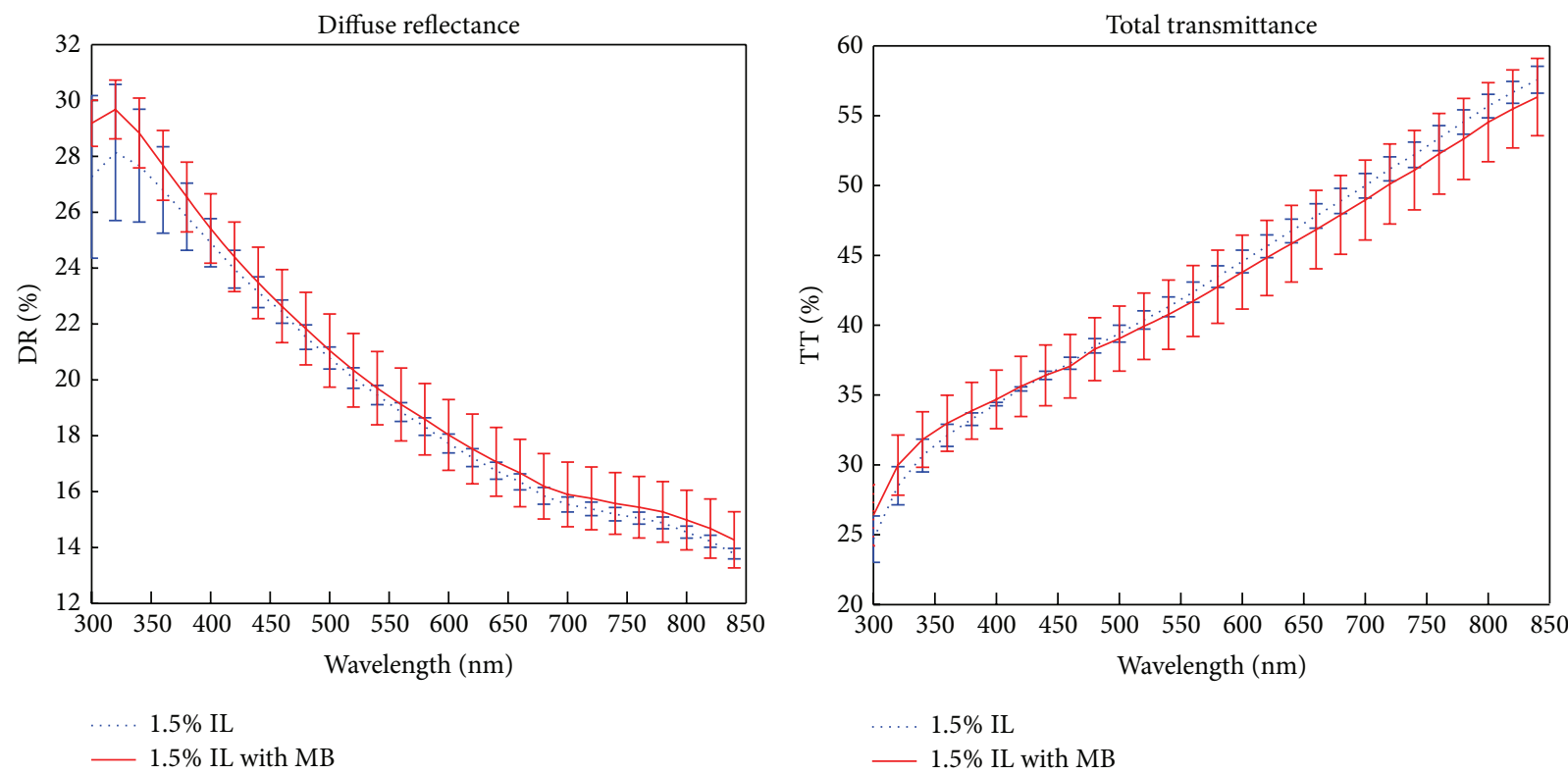

FIGURE 6: Diffuse reflectance (DR) and total transmittance (TT) of diluted intralipid (IL), $1.5 \%$ concentration, before and after adding Definity microbubbles.
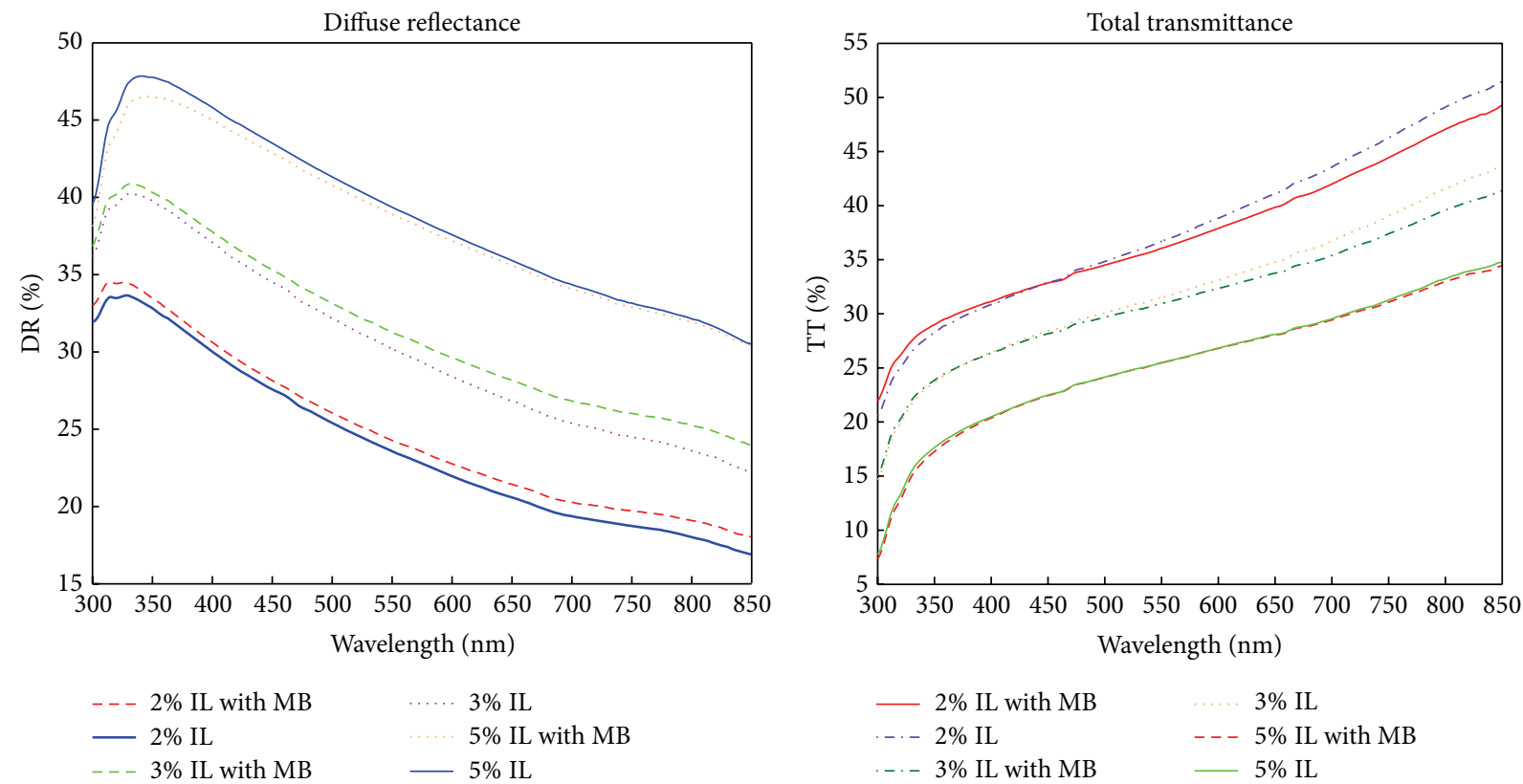

FIGURE 7: Diffuse reflectance (DR) and total transmittance (TT) of diluted intralipid (IL), $2 \%, 3 \%$, and $5 \%$ concentrations, before and after adding Definity microbubbles.

caused by microbubbles are depicted in a solid line. The absorption coefficient of intralipid phantom measured in the study (Figure 8 ) was higher compared to those reported in the literature: $0.01(1 / \mathrm{cm})$ at $500 \mathrm{~nm}$ [19]. This may be due to integrating sphere corrections and lost light during the measurements [13]. The lost light is considered part of absorption and consequently overestimating $\mu_{a}$. The results indicate that both $\mu_{a}$ and $\mu_{s}^{\prime}$ increase significantly after adding microbubbles to the phantom intralipid solution at $0.5 \%, 1 \%$, and $1.5 \%$ (Figures $10-12$ ). The reduced scattering coefficient decreased with the wavelength; at wavelengths of $700 \mathrm{~nm}$ and longer, the absorption coefficient is lower 

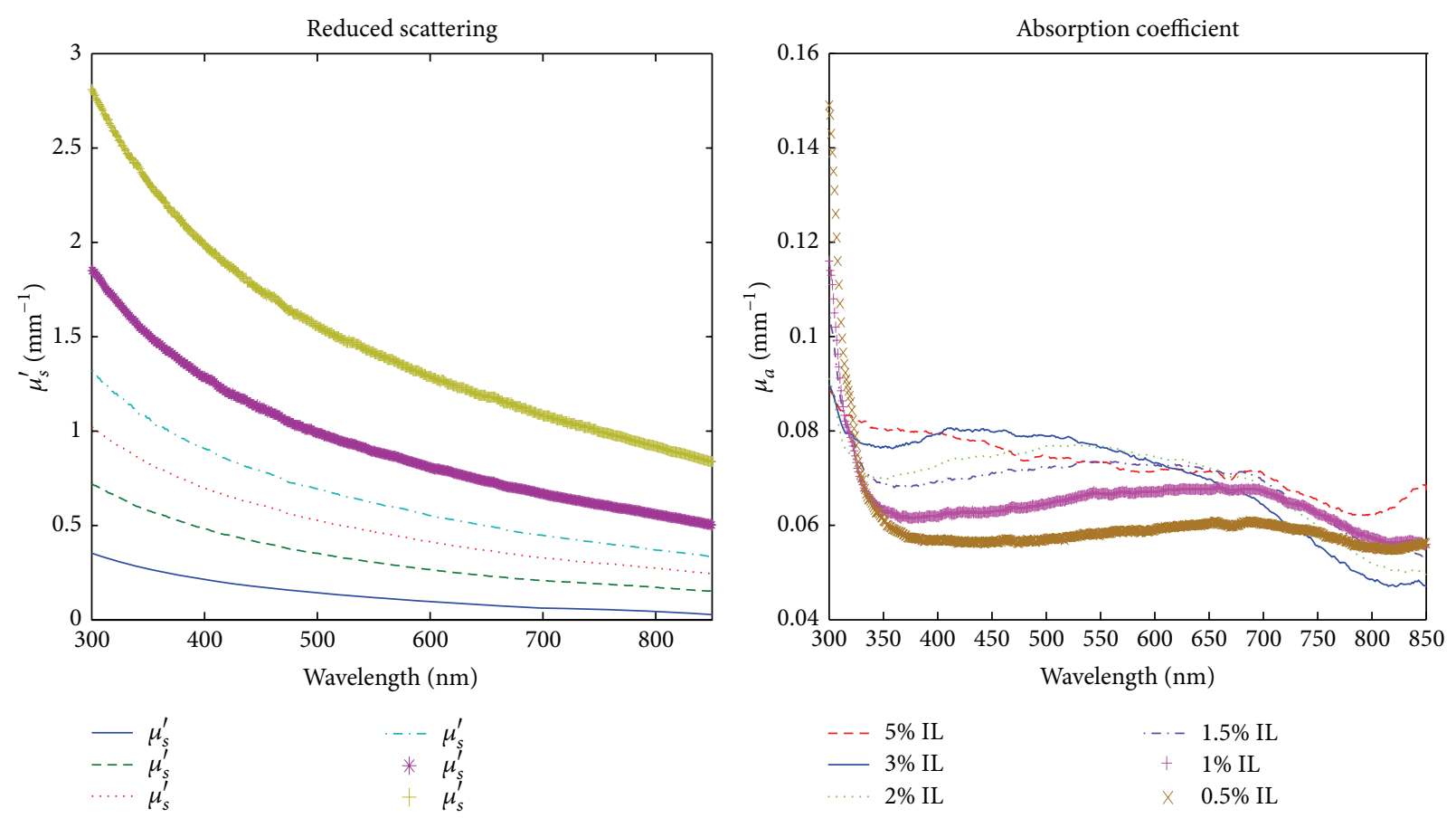

FigURE 8: Reduced scattering and absorption coefficient of all concentrations of intralipid.

than 0.1. However, at higher intralipid concentrations, the intrinsic optical properties were not significantly different in the presence and absence of microbubbles (Figure 13). The absorption coefficient $\mu_{a}$ and the reduced scattering coefficient $\mu_{s}^{\prime}$ increased in the presence of microbubbles. The growth of the scattering coefficient was more significant (1.35 fold at $300 \mathrm{~nm}$ and 6 -fold at $850 \mathrm{~nm}$ ) compared to the absorption coefficient (1.12 fold maximum across the spectrum) for the $0.5 \%$ intralipid phantom.

The scattering and absorbing particles of intralipid are soybean oil and water. It has been stated that water is the significant light absorber of intralipid in near infrared light for the wavelength range over $950 \mathrm{~nm}$ which is beyond the range we explored for the study [4]. It is found that in the red and far-red regions of the spectrum, both water and soybean oil contribute to the absorption while in the blue region, the absorption is primarily due to soybean oil [14].

\section{Discussion and Conclusions}

The presence of microbubbles in a scattering-mimicking tissue phantom significantly changed its optical properties in the 300-850 nm wavelength range. The scattering coefficient increased in the presence of microbubbles within the biological tissue transparency window where tissue absorption is minimal [20]. Microbubbles affected the intrinsic optical properties of the tissue phantom-both scattering and absorption. The microbubbles introduced an extrarefractive index mismatch that increased the probability of scattering

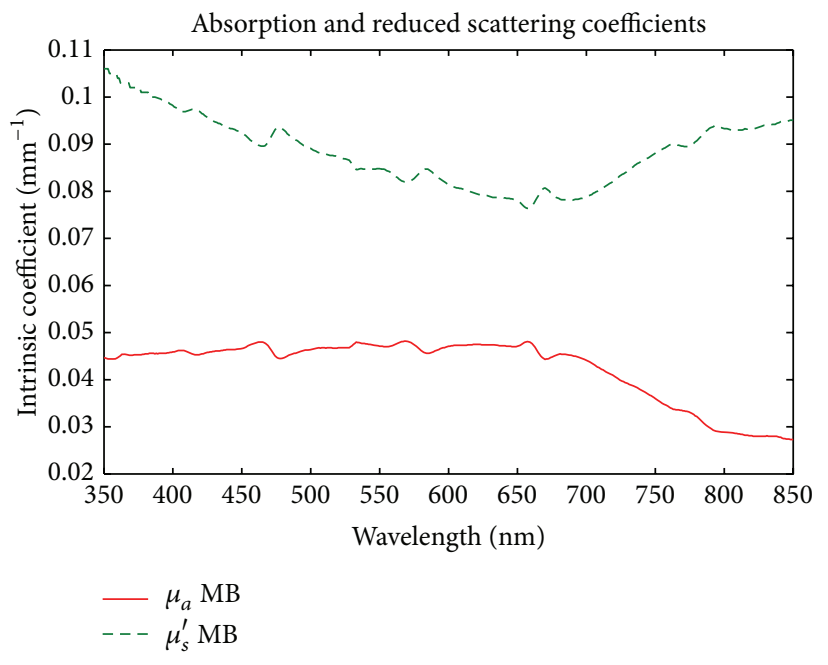

Figure 9: Reduced scattering and absorption coefficient for microbubbles diluted in deionized water (without intralipid).

events. The increase in light attenuation events is associated with the presence of microbubbles. Adding microbubbles changes the intrinsic optical properties of the tissue phantom not only for scattering but also for the absorption. The effect of microbubbles on reduced scattering and absorption 

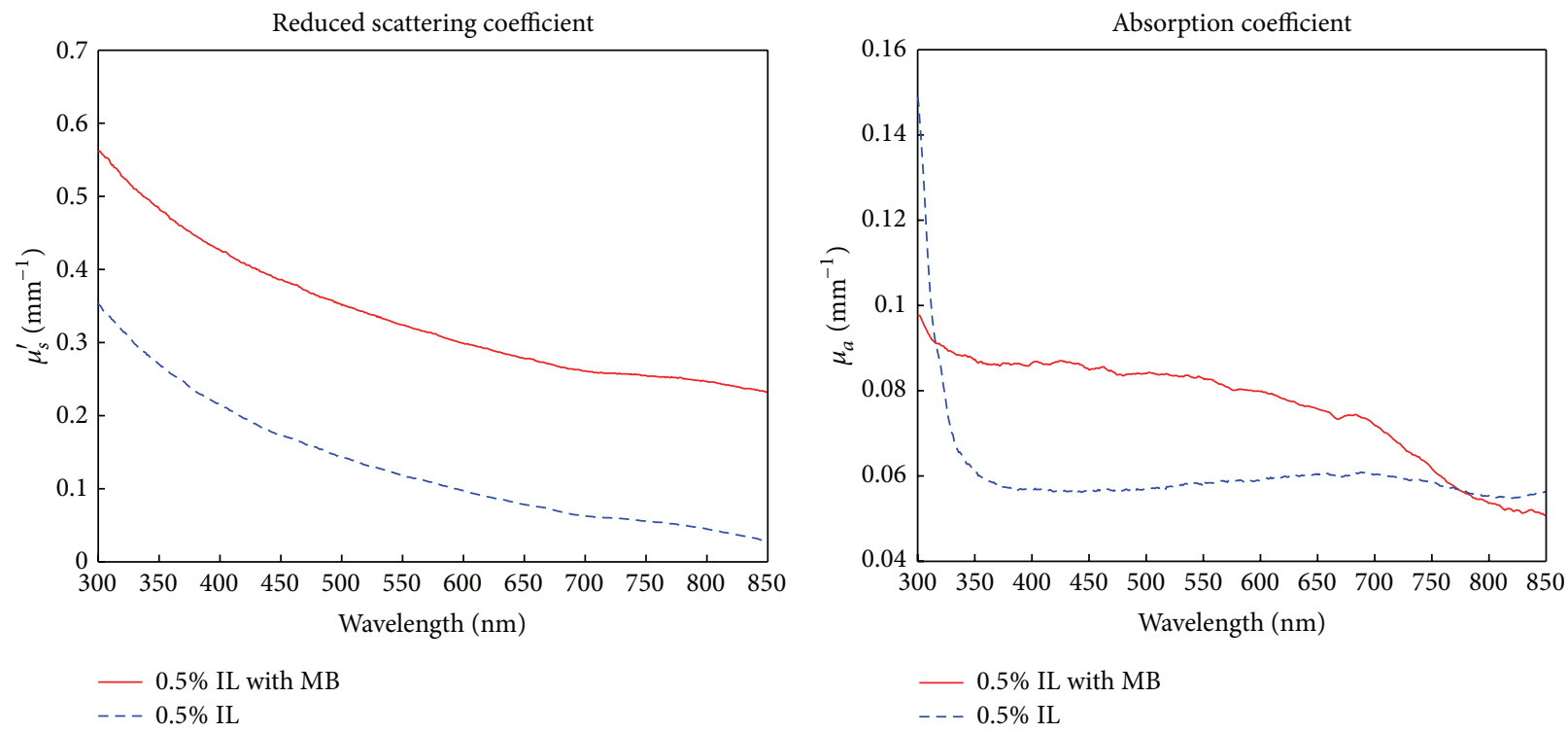

FIGURE 10: Absorption coefficient $\left(\mu_{a}\right)$ and reduced scattering coefficient $\left(\mu_{s}^{\prime}\right)$ of diluted intralipid (IL), $0.5 \%$ concentration, before and after adding Definity microbubbles.
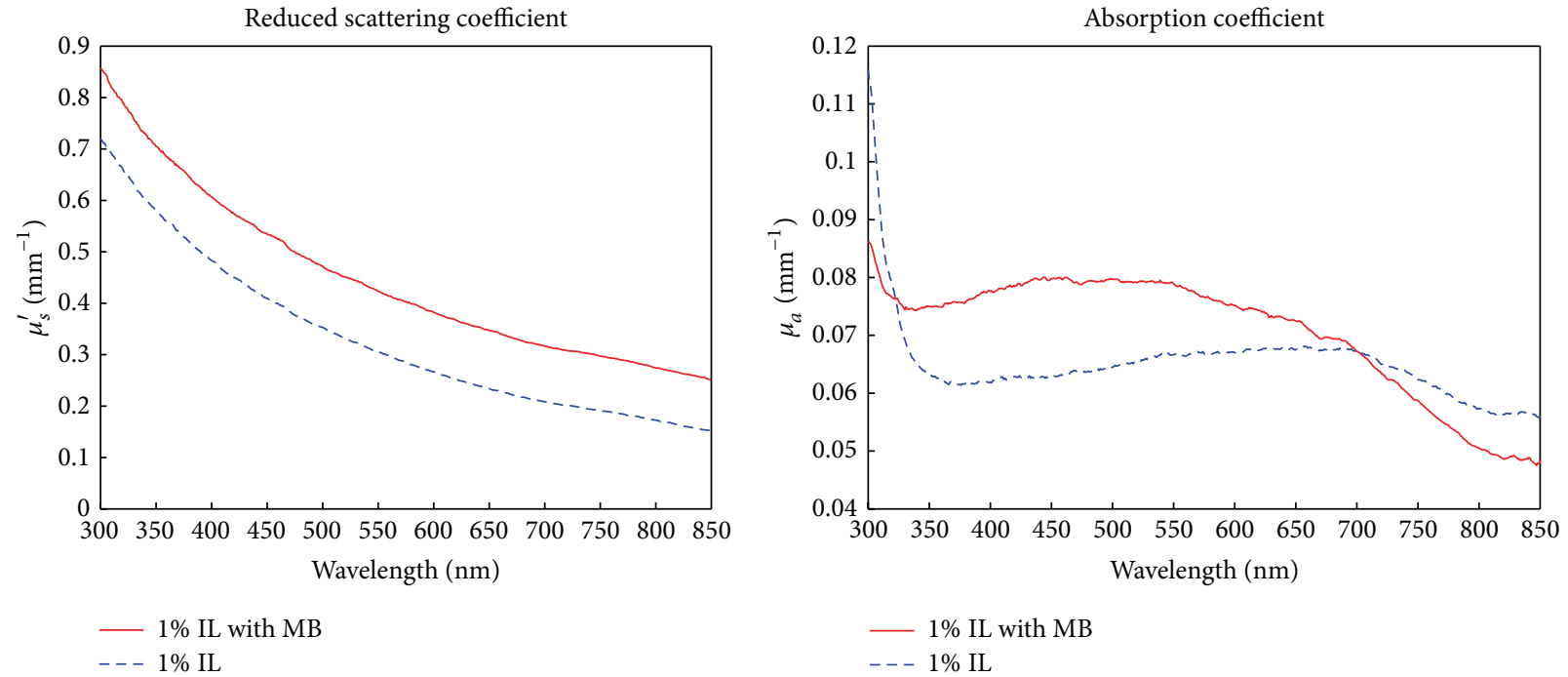

FIGURE 11: Absorption coefficient $\left(\mu_{a}\right)$ and reduced scattering coefficient $\left(\mu_{s}^{\prime}\right)$ of diluted intralipid (IL), $1 \%$ concentration, before and after adding Definity microbubbles.

coefficients decreased at longer wavelengths. In addition, at higher concentrations of intralipid $(>1.5 \%)$, the effect of adding microbubbles is significantly reduced. The scattering properties of most biological tissues can be approximated at intralipid concentrations from $0.5 \%$ to $5.0 \%$ [12], indicating that the enhancement of the backscattering and reflectance due to the presence of microbubbles $(2.5 \% \mathrm{v} / \mathrm{v})$ can be potentially employed for improving optical imaging diagnostics. In contrast-enhanced ultrasound imaging, the dose of microbubbles depends on the contrast agent characteristics and mode of administration. The microbubbles can be administered through infusion or bolus injection. Bolus injection is a delivery of an agent in a single bolus usually over a few seconds as opposed to infusion where the agent is delivered over a longer period and lower flow rate (e.g., saline bag infusion). A dose of $2.5 \%$ can be potentially achieved when microbubbles are administered through a bolus injection [21]. 

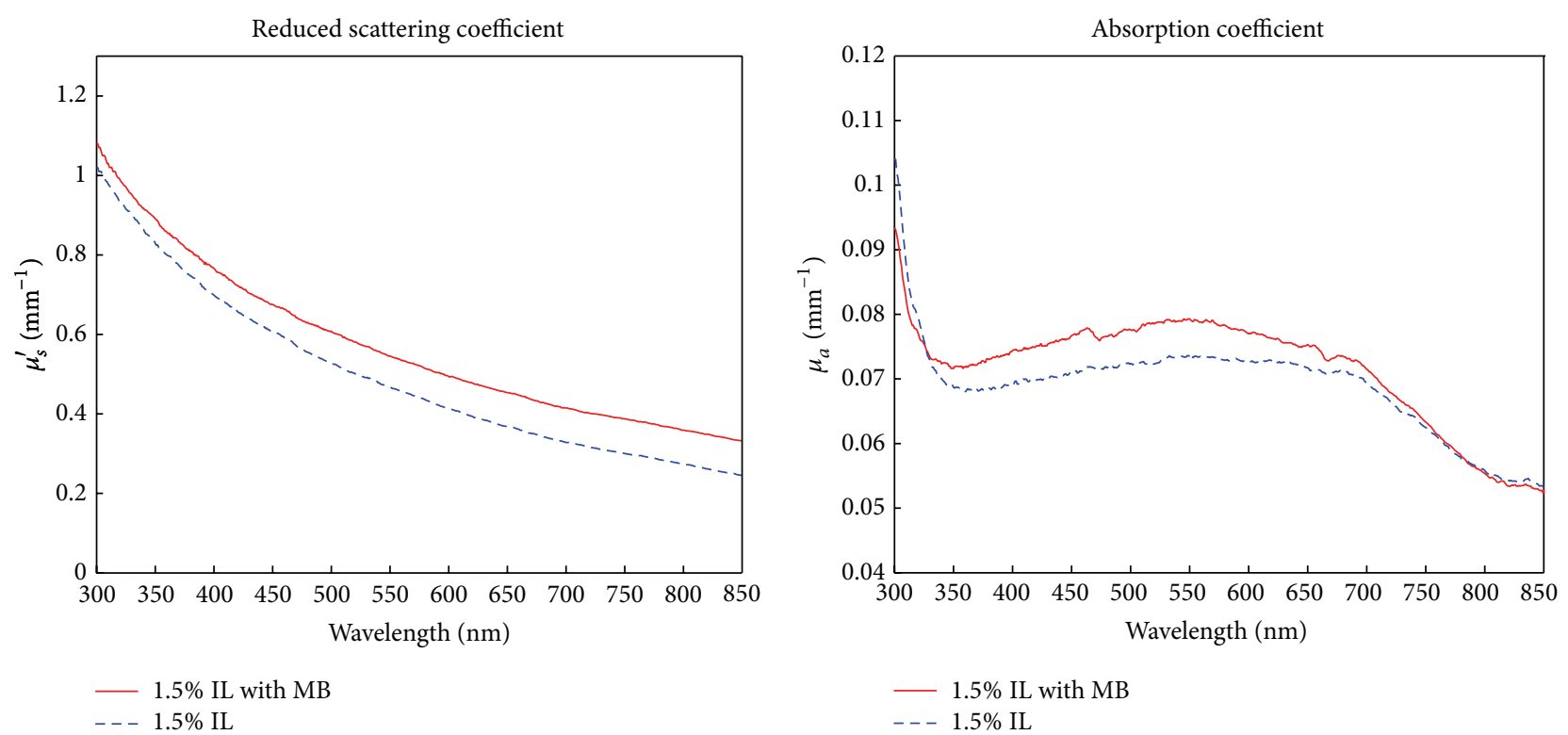

FIGURE 12: Absorption coefficient $\left(\mu_{a}\right)$ and reduced scattering coefficient $\left(\mu_{s}^{\prime}\right)$ of diluted intralipid (IL), $1.5 \%$ concentration, before and after adding Definity microbubbles.
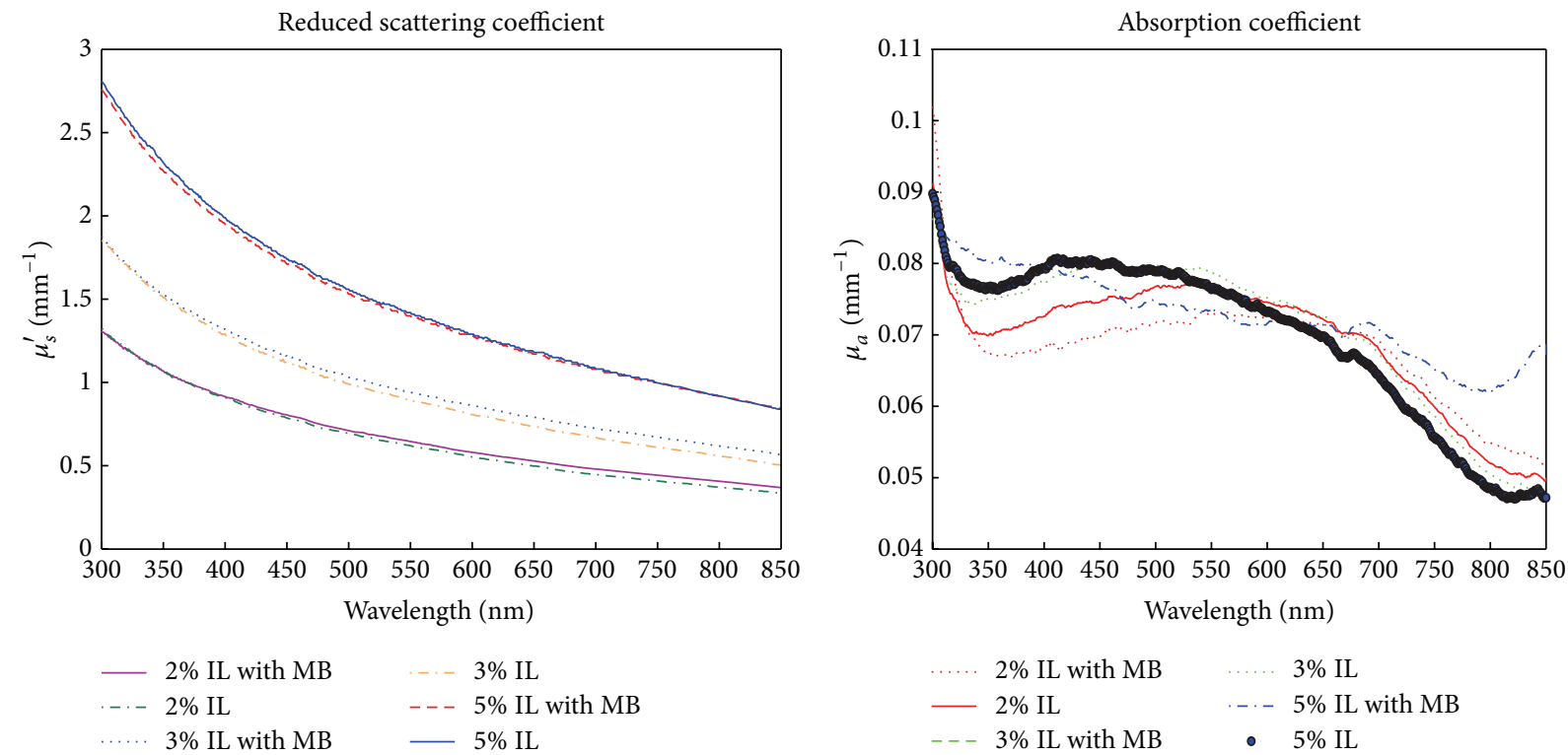

FIGURE 13: Absorption coefficient $\left(\mu_{a}\right)$ and reduced scattering coefficient ( $\left.\mu_{s}^{\prime}\right)$ of diluted intralipid (IL), $2 \%$, 3\%, and 5\% concentrations, before and after adding Definity microbubbles.

\section{Conflict of Interests}

The authors declare that there is no conflict of interests regarding the publication of this paper.

\section{Acknowledgments}

The authors thank Dr. Carl Kumaradas, Physics Department, Ryerson University, Toronto, for his advice and assistance and Martin Hohmann, University Erlangen-Nuremberg, Germany, for useful discussions. The authors gratefully acknowledge the financial support (in part) provided by the Ryerson University Health Research Grant, NSERC Personal Discovery Grant, Canada and Erlangen Graduate School in Advanced Optical Technologies (SAOT), and German National Science Foundation (DFG), Germany, in the framework of the excellence initiative. Thanks are given to the Keenan Research Centre of the LKS Knowledge Institute, St. Michael Hospital, Toronto, and Erlangen Graduate 
School in Advanced Optical Technologies (SAOT), University Erlangen-Nuremberg, Germany.

\section{References}

[1] S. R. Sirsi and M. A. Borden, "Microbubble compositions, properties and biomedical applications," Bubble Science, Engineering and Technology, vol. 1, no. 1-2, pp. 3-17, 2009.

[2] F. Saglimbeni, S. Bianchi, G. Bolognesi, G. Paradossi, and R. di Leonardo, "Optical characterization of an individual polymer-shelled microbubble structure via digital holography," Soft Matter, vol. 8, no. 34, pp. 8822-8825, 2012.

[3] J. J. Kwan and M. A. Borden, "Lipid monolayer collapse and microbubble stability," Advances in Colloid and Interface Science, vol. 183-184, pp. 82-99, 2012.

[4] H. J. van Staveren, C. J. Moes, J. van Marie, S. A. Prahl, and M. J. van Gemert, "Light scattering in intralipid-10\% in the wavelength range of 400-1100 nm," Applied Optics, vol. 30, no. 31, pp. 4507-4514, 1991.

[5] A. P. Dhawan, B. d'Alessandro, and X. Fu, "Optical imaging modalities for biomedical applications," IEEE Reviews in Biomedical Engineering, vol. 3, pp. 69-92, 2010.

[6] T. M. Lee, A. L. Oldenburg, S. Sitafalwalla et al., "Engineered microsphere contrast agents for optical coherence tomography," Optics Letters, vol. 28, no. 17, pp. 1546-1548, 2003.

[7] M. Douas, M. I. Marqués, and P. A. Serena, "Optical image contrast enhancement in near-field optics induced by water condensation," Ultramicroscopy, vol. 135, pp. 50-55, 2013.

[8] P. A. Dijkmans, L. J. M. Juffermans, R. J. P. Musters et al., "Microbubbles and ultrasound: from diagnosis to therapy," European Journal of Echocardiography, vol. 5, no. 4, pp. 245-256, 2004.

[9] CRCnetBASE, Biomedical Photonics Handbook, CRC Press, 2003.

[10] A. E. Profio, "Light transport in tissue," Applied Optics, vol. 28, no. 12, pp. 2216-2222, 1989.

[11] A. J. Welch and M. J. C. Gemert, Eds., Optical-Thermal Response of Laser-Irradiated Tissue, chapter 8, Springer, Dordrecht, The Netherlands, 2011.

[12] S. T. Flock, S. L. Jacques, B. C. Wilson, W. M. Star, and M. J. C. van Gemert, "Optical properties of intralipid: a phantom medium for light propagation studies," Lasers in Surgery and Medicine, vol. 12, no. 5, pp. 510-519, 1992.

[13] S. Prahl, "Optical Property Measurements Using the Inverse Adding-Doubling Program".

[14] P. D. Ninni, F. Martelli, and G. Zaccanti, "Intralipid: towards a diffusive reference standard for optical," Physics in Medicine and Biology, vol. 56, no. 2, pp. N21-N28, 2011.

[15] S. K. Cool, B. Geers, S. Roels et al., "Coupling of drug containing liposomes to microbubbles improves ultrasound triggered drug delivery in mice," Journal of Controlled Release, vol. 172, no. 3, pp. 885-893, 2013.

[16] "UV-2600, UV-2700 UV-VIS Spectrophotometer: SHIMADZU (Shimadzu Corporation)," http://www.shimadzu.com/an/ uv/uv2600_2700.html.

[17] S. Prahl, "Everything i think you should know about inverse adding-doubling," vol. 2197, 2011.

[18] M. L. Dong, K. G. Goyal, B. W. Worth et al., "Accurate in situ measurement of complex refractive index and particle size in intralipid emulsions," Journal of Biomedical Optics, vol. 18, no. 8, Article ID 87003, 2013.
[19] “Optical Properties of Intralipid," http://omlc.ogi.edu/spectra/ intralipid/index.html.

[20] R. R. Anderson and J. A. Parrish, "The optics of human skin," Journal of Investigative Dermatology, vol. 77, no. 1, pp. 13-19, 1981.

[21] T. A. Fritz, E. C. Unger, G. Sutherland, and D. Sahn, "Phase I clinical trials of MRX-115: a new ultrasound contrast agent," Investigative Radiology, vol. 32, no. 12, pp. 735-740, 1997. 

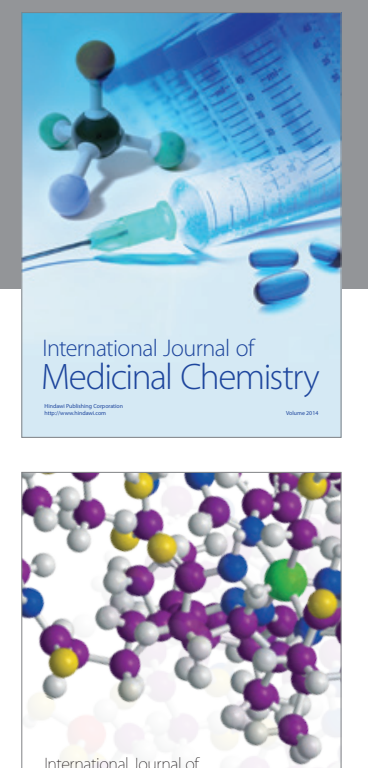

\section{Carbohydrate} Chemistry

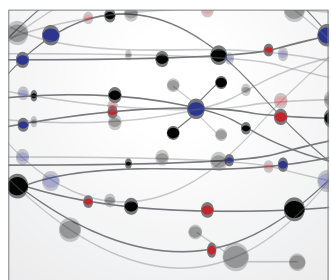

The Scientific World Journal
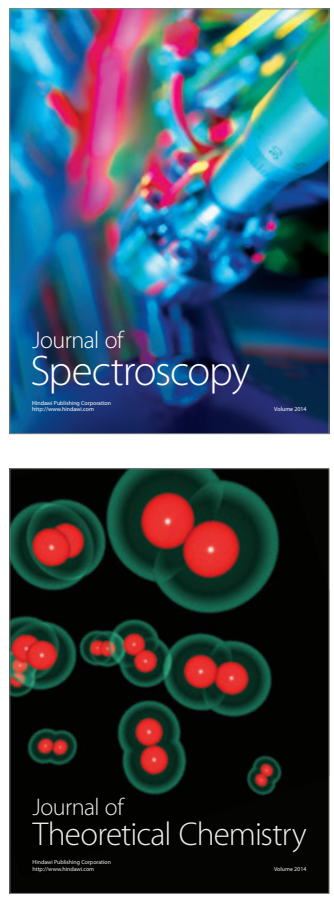
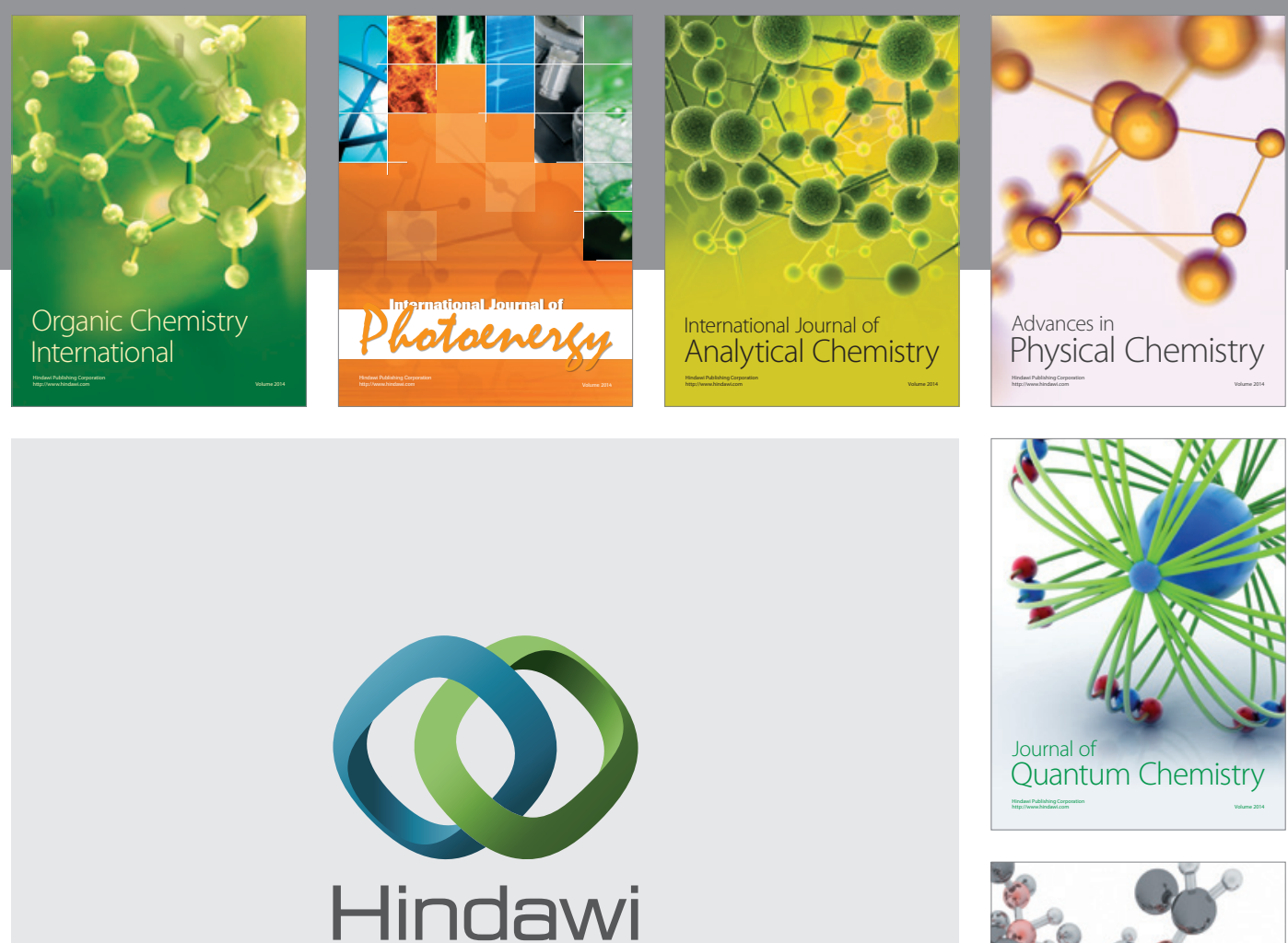

Submit your manuscripts at

http://www.hindawi.com

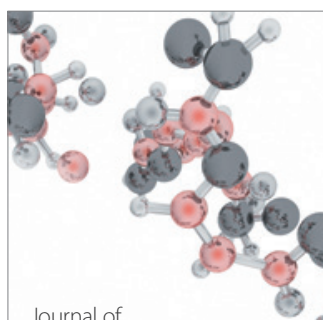

Analytical Methods

in Chemistry

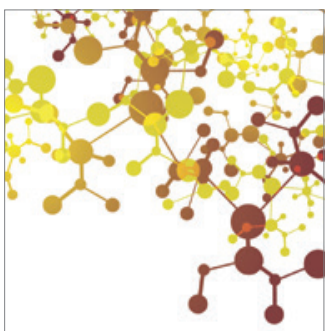

Journal of

Applied Chemistry

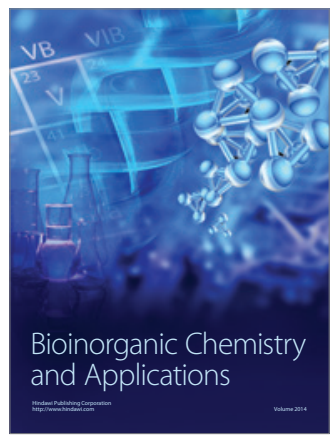

Inorganic Chemistry
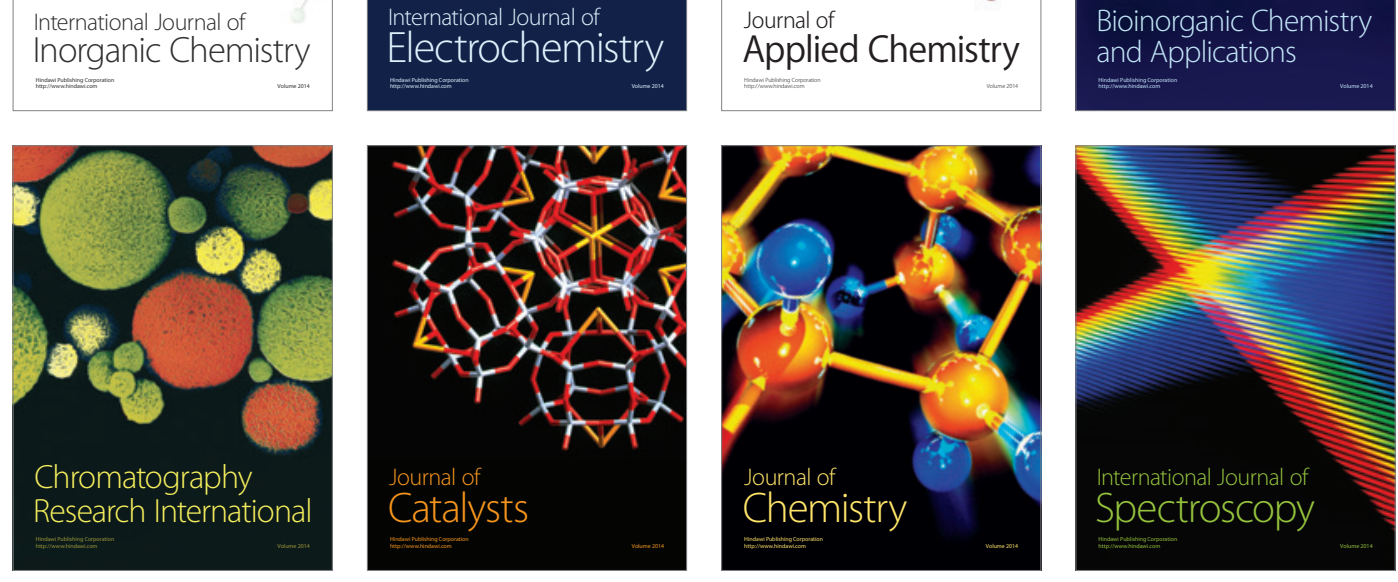